\title{
Use of the star-triangle transformation for the application of differential real-space renormalization-group theory
}

\author{
Wim van Saarloos \\ Instituut Lorentz, Nieuwsteeg 18, 2311-SB Leiden, The Netherlands, \\ and Bell Laboratories, Murray Hill, New Jersey 07974*
}

(Received 7 January 1983)

\begin{abstract}
When differential real-space renormalization-group theory was proposed by Hilhorst, Schick, and van Leeuwen, they suggested that their approach could only be applied to lattice models for which a star-triangle transformation exists. However, differential renormalization-group equations for the square Ising model have recently been proposed whose derivation does not involve the star-triangle transformation. We show that the latter equations are not exact renormalization-group equations by an analysis that reveals some essential limitations of the present formulation of differential real-space renormalization. We investigate the structure of the renormalization-group flow equations obtained in this method and uncover a strong property of these equations that simplifies the calculations in actual applications of the theory. However, the status and implications of this property, which embodies the crux of the theory, are not yet fully understood.
\end{abstract}

\section{INTRODUCTION}

In the well-known block-spin renormalizationgroup methods, ${ }^{1}$ one groups together a whole block of $l^{d}$ site spins (where $d$ is the dimensionality and $l$ is the linear dimension of the block in units of the lattice parameter). This block of site spins is then essentially represented by one single cell spin so that the number of degrees of freedom on the renormalized lattice is only a finite fraction $l^{-d}$ of the number of degrees of freedom on the original site spin lattice. In such cases each successive renormalization step can be represented by a finite jump in the parameter space. The observation that one will obtain flow equations in differential form for a renormalization transformation in which only an infinitesimal fraction of the spins is thinned out underlies the so-called differential real-space renormalization-group (DRSRG) theory of Hilhorst, Schick, and van Leeuwen ${ }^{2}$ (HSL). In their method a lattice $L$ of $(N+1)^{d}$ spins is mapped onto a similar lattice $L^{\prime}$ of $N^{d}$ spins, so that for large $N$ the renormalized lattice $L^{\prime}$ contains only a fraction $(N+1)^{d} / N^{d}-1 \approx d / N$ less than $L$, the original one. By taking the thermodynamic limit $N \rightarrow \infty$, the renormalization flow equations can then be cast in differential form.

HSL showed that this program can be carried out explicitly for an Ising model on a triangular lattice. They thus obtained exact DRSRG equations for the triangular Ising model. The linearized flow around the fixed point they found was consistent with the well-known exact results for the Ising model.

As was noted already by HSL, the so-called startriangle transformation (STT) for Ising models, ${ }^{3}$ illustrated in Fig. 1(a), appeared to be an indispensable tool in DRSRG. Indeed, later applications of DRSRG to the $d$-dimensional Gaussian model,,$^{4-6}$

(a)

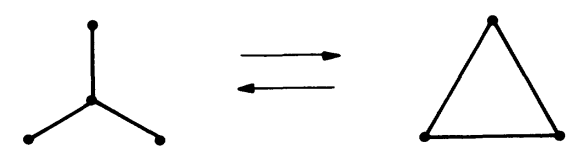

(b)

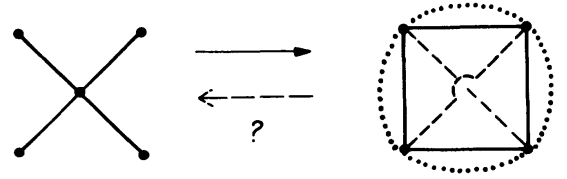

FIG. 1. (a) STT transformation for the Ising model converts the star of three nearest-neighbor interactions (solid lines) to a triangle of interactions. The inverse transformation always exists. (b) The star-square transformation converts the star of four interactions to a square with nearest-neighbor, next-nearest-neighbor (dashed diagonal lines), and a four-spin interaction (dotted circle). The inverse transformation generally does not exist. 
the $q$-state Potts model in the limit $q \rightarrow 0,{ }^{7}$ and even to the square Ising model $^{8}$ relied heavily on the STT or a generalization thereof. All these applications therefore seem to substantiate HSL's surmise that DRSRG is useful only for lattice models to which the STT can be applied. In this light it appears worthwhile to investigate a recent suggestion ${ }^{9}$ that the square Ising model can be analyzed within the framework of DRSRG without invoking the STT at all. In this paper we argue, however, that this proposed transformation is not a proper renormalization-group transformation, and more generally point out some of the inherent limitations of DRSRG, as presently implemented.

The problem with the suggested renormalizationgroup transformation is related to one that arises in certain block-spin transformations. As discussed, e.g., by van Leeuwen, ${ }^{10}$ block-spin methods that result from summing over part of the site spins $s_{\circ}$ while keeping a subset $s_{\bullet}$ of them fixed are often inconsistent with the known behavior of the correlation function of the model under investigation. Such transformations, often called decimation transformations, are defined in terms of the site spin Hamiltonian $H\left(s_{\bullet}, s_{\bigcirc}\right)$ (with interaction parameters $\left.K_{i}\right)$ and the renormalized Hamiltonian $H^{\prime}\left(s_{\bullet}\right)$ (interactions $K_{i}^{\prime}$ ) as

$$
e^{-\beta H^{\prime}\left(s_{\bullet}\right)}=\sum_{\left\{s_{\bigcirc}\right\}} e^{-\beta H\left(s_{\bullet}, s_{\bigcirc}\right)},
$$

where the summation is over the states of the spins $s_{\circ}$. If one denotes by $g\left(\vec{\rho},\left\{K_{i}\right\}\right)$ the correlation function of two spins $s_{\bullet}$ a distance $\vec{\rho}$ apart on the site spin lattice, then it follows immediately from Eq. (1) that

$$
g\left(\vec{\rho},\left\{K_{i}\right\}\right)=g\left(\vec{\rho} / l,\left\{K_{i}^{\prime}\right\}\right),
$$

where $l$ is the scaling length of the transformation. Equation (2) shows that for this transformation the correlation function is independent of the distance $\rho$ at the fixed point $K_{i}^{*}$ since $g\left(\vec{\rho},\left\{K_{i}^{*}\right\}\right)=g\left(\vec{\rho} / l,\left\{K_{i}^{*}\right\}\right)$. This result is in contradiction with the critical behavior of most lattice models that have a phase transition at finite temperature.

The above arguments do not immediately apply to DRSRG, since in this theory the interaction parameters are dependent on the position on the lattice, and the scaling of the length enters in a different way. After having summarized the essentials of DRSRG in Sec. II, we therefore extend the above ideas to the case of DRSRG by discussing the behavior of the correlation functions of spins that remain fixed during the transformation. As in the case of block-spin methods, the analysis rules out a certain class of transformations because of incon- sistencies, including the one proposed by Jezewski ${ }^{9}$; moreover, it will support the original suggestion of HSL (Ref. 2) that the STT is indeed essential in their formulation of DRSRG.

Though it is easy to assess why the approach of HSL cannot be implemented in certain cases, the reason why the theory is successful in other cases is, in our opinion, not fully understood. In fact, there is a simple but quite strong property of the general DRSRG flow equations that embodies the crux of the theory but whose status and implications are still unclear. Our analysis of the square Ising model ${ }^{8}$ was already based on this property, but its discussion was obscured by the detailed calculations. It is therefore rederived in a simpler and more general way in Sec. IV, and we hope that its clarification will indicate how the theory can be adapted so as to make it more generally applicable.

\section{RECAPITULATION OF DRSRG THEORY}

In this section we retrace the two main ingredients of DRSRG by recalling briefly the derivation of the equations for the triangular Ising model. ${ }^{11}$ The transformation of HSL consists of two steps (see Fig. 2):

I. A transformation of a triangular Ising lattice $L_{T}$ with $1 / 2 N(N+1)$ spins [closed circles, Fig. 2(a)] into a hexagonal lattice (Fig. 2(b)] and then back into a triangular lattice $\widetilde{L}_{T}$ of $1 / 2 N(N-1)$ spins [open circles, Fig. 2(c)]. In this step the STT as well as the inverse STT are employed.

II. A uniform rescaling of the coordinate system with respect to the center $O$ so as to make the new renormalized lattice $L_{T}^{\prime}$ [Fig. 2(d)] of the same size as the old one.

Steps I and II together form the basis of DRSRG theory. At this point it is worthwhile to recall the well-known fact that renormalization-group theory requires that each renormalization step be repeatable. This means that starting from a lattice $L$ one should be able to construct the whole renormalization sequence

$$
L \rightarrow L^{\prime} \rightarrow L^{\prime \prime} \rightarrow L^{\prime \prime \prime} \rightarrow \cdots,
$$

$L^{\prime \prime}$ being the renormalized of $L^{\prime}$, etc. In the transformation of HSL this requirement is fulfilled since steps Ia, Ib, and II of Fig. 2 can be performed for any given starting lattice. Indeed, it is instructive to summarize the renormalization-group transformations of HSL symbolically by

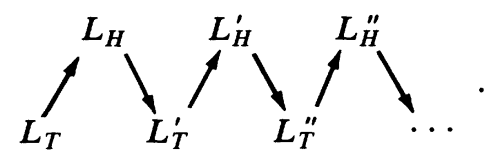




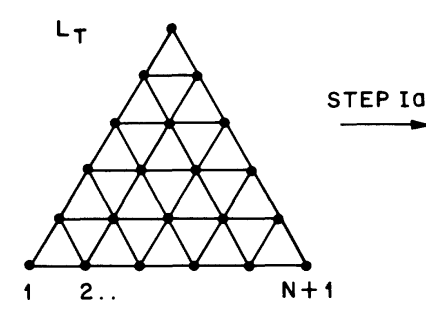

(a)

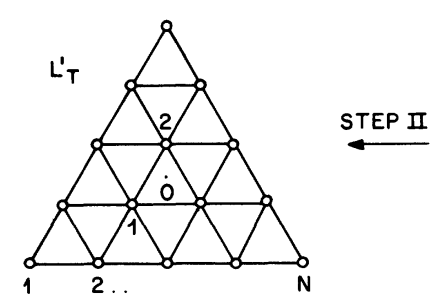

(d)

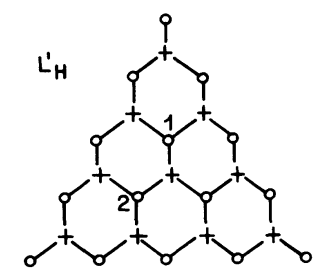

(e)

FIG. 2. Renormalization transformation of HSL for the triangular lattice. Units are such that the total length of an edge of $L_{T}$ in (a) is 1 , so that the lattice constant is $1 / N$. In step Ia the lattice $L_{T}$ is transformed into a hexagonal lattice $L_{H}$, using the inverse STT. In step Ib the STT is applied to the spins indicated by closed circles on $L_{H}$, yielding the lattice $\widetilde{L}_{T}$. Finally the renormalized lattice $L_{T}^{\prime}$ is obtained from $\widetilde{L}_{T}$ by an overall stretching. The next hexagonal lattice in the sequence is shown in (e).

The hexagonal lattice $L_{H}^{\prime}$ is also drawn in Fig. 2. Of course, the upper set of lattices $L_{H}, L_{H}^{\prime}, L_{H}^{\prime \prime}, \ldots$ constitutes equally well a proper renormalization-group transformation as does the set of triangular lattices $L_{T}, L_{T}^{\prime}, L_{T}^{\prime \prime}, \ldots$.

HSL observed that even if they started with homogeneous interactions $K_{i}$ on the lattice $L_{T}$ (homogeneous meaning independent of the position $\overrightarrow{\mathbf{r}}$ on the lattice), then on the lattice $L_{T}^{\prime}$ the renormalized interactions $K_{i}^{\prime}$ near the edge were in general quite different from those in the bulk. HSL therefore concluded that it was necessary to formulate their theory for lattices with inhomogeneous interactions. The analysis of such lattices simplifies if the spatial dependence of the interactions is slowly varying over distances of the order of $1 / N$. HSL could show that if the functions $K_{i}(\vec{r})$ also satisfy appropriate boundary conditions the difference between $K_{i}^{\prime}(\vec{r})$ and $K_{i}(\vec{r})$ is only of order $1 / N$ (the lattice parameter) everywhere on the lattice. In the thermodynamic limit $1 / N \equiv \delta t \rightarrow 0$ their renormalization-group equations therefore become of the form

$$
\begin{gathered}
\frac{\partial K_{i}(\overrightarrow{\mathrm{r}}, t)}{\partial t}=\sum_{j=1}^{3} \overrightarrow{\mathrm{D}}_{i j}\left(K_{1}(\overrightarrow{\mathrm{r}}, t), K_{2}(\overrightarrow{\mathrm{r}}, t) K_{3}(\overrightarrow{\mathrm{r}}, t)\right) \\
\cdot \vec{\nabla} K_{j}(\overrightarrow{\mathrm{r}}, t)-\overrightarrow{\mathrm{r}} \cdot \vec{\nabla} K_{i}(\overrightarrow{\mathrm{r}}, t) .
\end{gathered}
$$

These equations are defined on a triangle with edges of length 1. Equation (5) expresses $\partial K_{i} / \partial t$, the change in the $K_{i}$ by one renormalization step in the limit $N \rightarrow \infty$, in terms of two contributions. The first, involving $\vec{D}_{i j}$, results from the local restructuring of the lattice (step I). This restructuring is an identity transformation for homogeneous lattices $\left(\vec{\nabla} K_{i}=0\right)$, but yields changes of order $1 / N$ if $\vec{\nabla} K_{i} \neq 0$. Note that $\overrightarrow{\mathrm{D}}_{i j}$, for which HSL had an explicit expression, does not depend on $\vec{r}$ or the "time" $t$. This is due to the fact that step $I$ is the same everywhere on the lattice and during every repeated renormalization. The scaling of the coordinates (step II of DRSRG) does depend on the position $\vec{r}$ on the lattice, and it gives rise to the term $-\overrightarrow{\mathrm{r}} \cdot \vec{\nabla} K_{i}$ in Eq. (5).

As mentioned above, Eq. (5) must be supplemented by the boundary conditions which ensure that the transformation stays infinitesimal at the edges too. However, we will not specify these, since they play no role in the following discussion.

\section{BEHAVIOR OF THE CORRELATION FUNCTIONS IN THE DRSRG}

We will now extend van Leeuwen's ${ }^{10}$ argument concerning decimation transformations, summarized in the Introduction, to DRSRG. The analysis for DRSRG differs from the one for block-spin methods for two reasons. Firstly, as discussed above, one now deals with lattices with inhomogeneous interactions. Secondly, whereas in block-spin methods the distance $\vec{\rho}$ between spins, measured in units of the lattice parameter, decreases a factor $l$ upon each renormalization step, this distance remains unchanged in DRSRG, even though the spins are situated at a different position $\vec{r}$ after renormalization (see below).

We first analyze the behavior of the correlation function in the transformation of HSL. As shown in Fig. 2, the triangular lattice $L_{T}$ (spins $s_{\bullet}$ ) and $L_{T}^{\prime}$ (spins $s_{O}$ ) have no spins in common. However, the 
hexagonal lattices $L_{H}$ and $L_{H}^{\prime}$ do have the spins $s_{\bigcirc}$ in common. This does not lead to inconsistencies, since unlike the decimation transformations in block-spin methods summarized by (2), the spins $s_{\circ}$ all disappear in the next transformation to the lattice $L_{H}^{\prime \prime}$. It is nevertheless instructive to analyze how the analog of Eq. (2) would read if all lattices $L_{H}, L_{H}^{\prime}, L_{H}^{\prime \prime}, \ldots$, would have spins $s_{\circ}$ in common. Consider, e.g., the spins $s_{\circ}$ labeled 1 and 2 on $L_{H}$ and $L_{H}^{\prime}$. On both lattices they are next-nearest neighbors, and their distance in units of the lattice parameter is therefore the same on $L_{H}$ and $L_{H}^{\prime}$. Their position is changed, however. If we denote their center on $L_{H}$ by $\overrightarrow{\mathrm{r}}_{c}$, then their center on $L_{H}^{\prime}$ is $\vec{r}_{c}(1+1 / N)$. We therefore get for the correlation function $g_{12}$ of these spins [cf. Eq. (2)]

$$
g_{12}\left(\left\{K_{i}^{h}(\overrightarrow{\mathrm{r}})\right\} ; \overrightarrow{\mathrm{r}}_{c}\right)=g_{12}\left(\left\{K_{i}^{h^{\prime}}(\overrightarrow{\mathrm{r}})\right\} ; \overrightarrow{\mathrm{r}}_{c}(1+1 / N)\right) \text {. }
$$

Here $K_{i}^{h}(\overrightarrow{\mathrm{r}})$ and $K_{i}^{h^{\prime}}(\overrightarrow{\mathrm{r}})$ denote the interaction parameters on the hexagonal lattices $L_{H}$ and $L_{H}^{\prime}$, respectively. The correlation functions in Eq. (6) depend on the interaction parameters on all positions $\vec{r}$. However, as was discussed for the linear Ising chain by van Saarloos et al., ${ }^{13}$ one can make a connection with the correlation function of homogeneous Ising models by assuming that $g_{12}$ only depends on $\overrightarrow{\mathrm{r}}_{c}$ through the values of the $K_{i}^{h}(\overrightarrow{\mathrm{r}})$ near $\overrightarrow{\mathrm{r}}_{c}$, and is independent of the gradients $\vec{\nabla} K_{i}^{h}$ (this assumption is investigated in more detail in Sec. IV). Following the ideas of DRSRG discussed in Sec. II, one may then expand the interaction parameters in $g_{12}$ around their values at $\vec{r}_{c}$ and take the usual limit $N \rightarrow \infty$. In cases that the spins $s_{\circ}$ do survive in successive renormalization steps, one then finds from Eq. (6) with the above assumptions

$$
\begin{aligned}
& \left.\sum_{i=1}^{3} \frac{\partial g_{12}\left(K_{1}^{h}, K_{2}^{h}, K_{3}^{h}\right)}{\partial K_{i}^{h}}\right|_{K_{i}^{h}=K_{i}^{h}\left(\overrightarrow{\mathrm{r}}_{c}, t\right)} \\
& \times\left(\frac{\partial K_{i}^{h}\left(\overrightarrow{\mathrm{r}}_{c}, t\right)}{\partial t}+\overrightarrow{\mathrm{r}}_{c} \cdot \vec{\nabla} K_{i}^{h}\left(\overrightarrow{\mathrm{r}}_{c}, t\right)\right)=0 .
\end{aligned}
$$

This equation expresses the fact that the change of these particular correlation functions under renormalization would be due only to the change in the interaction parameters caused by the scaling of the coordinate system in step II. Note that for the case of Fig. 2 the second term between brackets in (7) never vanishes. This is due to the fact that there are no spins $s_{O}$ on $L_{H}$, whose center $\overrightarrow{\mathrm{r}}_{c}$ coincides with the origin $O$ [see Fig. 2(b)], ${ }^{14}$ so that the center of the pair of spins $s_{O}$ is always on a different place on $L_{H}^{\prime}$ than it was on $L_{H}$.
Let us consider what would happen if there would exist pairs of spins $s_{\circ}$ on $L_{H}, L_{H}^{\prime}, L_{H}^{\prime \prime}, \ldots$, for which $\vec{r}_{c}=0$. For those, Eq. (7) would have read instead

$$
\begin{gathered}
\sum_{i=1}^{3} \frac{\partial g\left(K_{i}^{h}(\overrightarrow{0}, t), K_{2}^{h}(\overrightarrow{0}, t), K_{3}^{h}(\overrightarrow{0}, t)\right)}{\partial K_{i}^{h}(\overrightarrow{0}, t)} \frac{\partial K_{i}(\overrightarrow{0}, t)}{\partial t}=0 \\
\text { if } \overrightarrow{\mathrm{r}}_{c}=\overrightarrow{0}
\end{gathered}
$$

which implies that the correlation function of pairs for which $\overrightarrow{\mathrm{r}}_{c}=0$ remains always unchanged under renormalization. That is, of course, in contradiction with the general picture of renormalization-group theory that the correlation function changes and that the correlation length decreases as one moves away from criticality. The above discussion may therefore be summarized by stating that DRSRG can never be exact if both the original and all the renormalized lattices have a sublattice in common that contains spins whose center remains unchanged under the rescaling of the coordinates of step II.

As an application of these considerations, we now investigate the proposed transformation for the square lattice, 9 based on the so-called "star-square" transformation. ${ }^{15,16}$ The latter is illustrated in Fig. 1(b), and results from summing over the states of the Ising spin on the center of the star. This generates nearest-neighbor, next-nearest-neighbor, and a four-spin interaction between the remaining spins at the corners. As these seven interaction parameters all depend on the four nearest-neighbor interaction parameters of the star, they are not all independent. Consequently, while it is always possible to perform a star-square transformation, the inverse transformation is only possible in special cases in which the nearest-neighbor, next-nearest neighbor, and fourspin interaction satisfy certain relations.

Consider now the square Ising lattice of Fig. 3(a) with nearest-neighbor interactions only. By summing over the spins $s_{\bullet}$, the star-square transformation yields the lattice $L_{s}^{1}$ of Fig. 3(b), while summation over the spins $s_{+}$of $L_{s}$ yields the lattice $L_{s}^{2}$ of Fig. 3(c). This transformation symbolically reads<smiles>[Te][Te]</smiles>

To arrive at a renormalization-group transformation along these lines, (9) does not suffice since we must construct the whole renormalization sequence such as in (4). This means that it should be possible to transform $L_{s}^{2}$ of Fig. 3(c) into another smaller lattice such as in Fig. 3(d). If that could be done, the scaled lattice of Fig. 3(e) would be the renormalized of $L_{s}$, and we would apparently have obtained a 


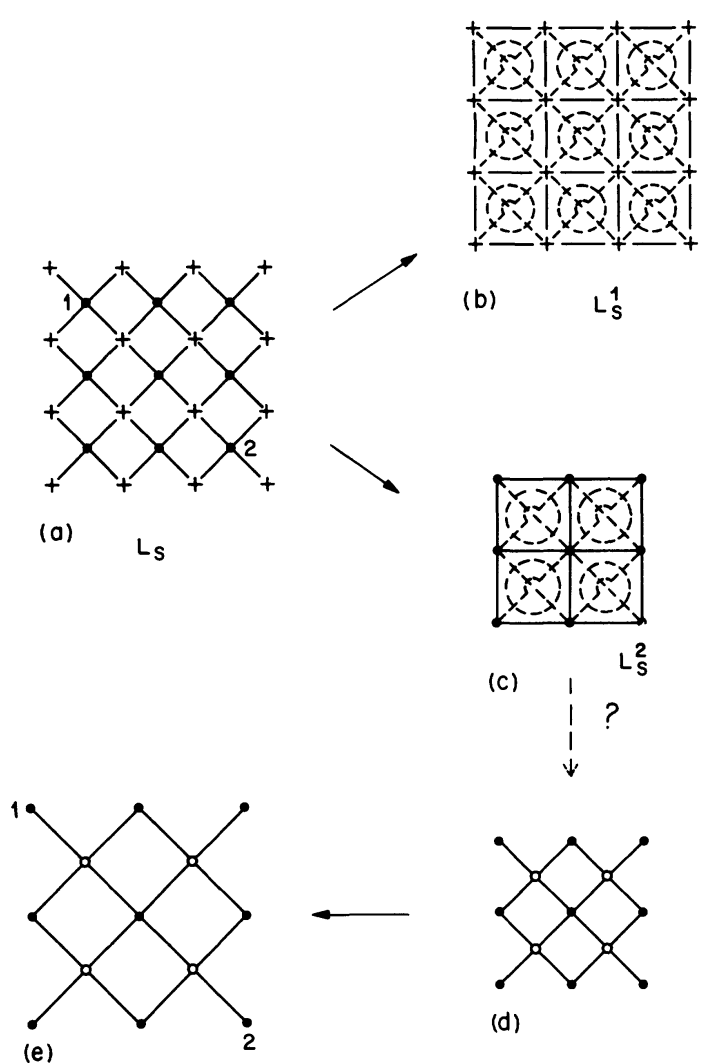

FIG. 3. Transformation for the square Ising model discussed in the text.

DRSRG transformation for the square lattice. However, the crucial transformation from the lattice of Fig. 3(c) to the one of 3(d) can, in general, not be performed, since the inverse of the star-square transformation does not exist. ${ }^{17}$ Moreover, our previous argument immediately shows that the very existence of such a transformation is, in fact, in contradiction with the renormalization-group picture itself. For if the transformation to the lattice 3(d) and 3(e) could exist, then one finds that the sublattice of spins indicated with closed circles reappears after renormalization in every step. On this sublattice there are spins whose center coincides with the origin (e.g., the spins 1 and 2 in the figure). As we discussed above, Eq. (8) applies to such spins, implying that the transformation as proposed in Ref. 9 cannot lead to a proper (exact) DRSRG scheme.

Our analysis fully supports the original suggestion of HSL that the STT is the essential tool for DRSRG. Indeed, the basic dilemma of the theory is that, on the one hand, one should compare lattices of nearly the same size, while on the other hand, these lattices may not have pairs of spins in common whose center has not been shifted by the scaling. Moreover, every lattice should have two related lattices, a "parent" and a "child." For the case con- sidered by HSL, two related lattices exist because the STT can be applied in upward-pointing triangles (step Ia of Fig. 2) as well as in downward-pointing triangles (step Ib). This, however, is possible because only nearest-neighbor interactions are involved. The fact that generalizations of the STT generally also give rise to higher-order interactions is the main problem preventing their use in DRSRG.

We have based our interpretation of Eq. (6) on the assumption that the correlation function is independent of the gradients $\vec{\nabla} K_{i}$. In all applications of the theory to date, these gradients are eliminated in a similar way at some point. In the last section we analyze when this is possible.

\section{ELIMINATION OF THE GRADIENTS FROM THE LINEAR FLOW}

In this section we suppose that a proper DRSRG transformation has been found for a given model with interaction parameters $K_{1}, \ldots, K_{n}$ and discuss one of the limitations revealed by the structure of the resulting renormalization-group flow equations,

$$
\begin{aligned}
\frac{\partial K_{i}(\overrightarrow{\mathrm{r}}, t)}{\partial t}=\sum_{j=1}^{n} & \overrightarrow{\mathrm{D}}_{i j}\left(K_{1}(\overrightarrow{\mathrm{r}}, t), \ldots, K_{n}(\overrightarrow{\mathrm{r}}, t)\right) \\
& \cdot \vec{\nabla} K_{j}(\overrightarrow{\mathrm{r}}, t)-\overrightarrow{\mathrm{r}} \cdot \vec{\nabla} K_{i}(\overrightarrow{\mathrm{r}}, t) .
\end{aligned}
$$

This generalization of Eq. (5) to the case of $n$ interaction parameters is the prototype DRSRG flow equation. ${ }^{18}$ We will assume that these equations are valid in some domain in $d$ dimensions. As before, we will not specify the boundary conditions to be satisfied by the functions $K_{i}(\overrightarrow{\mathrm{r}}, t)$, as they play no role in the subsequent analysis.

Not only does DRSRG deal with lattices with inhomogeneous interactions, but the inhomogeneities are, in fact, the basic feature of the theory since the flow in the parameter space (an infinite dimensional function space) is driven by the gradients in the functions $K_{i}(\vec{r})$. It is therefore rather surprising that DRSRG theory can be used at all to calculate the exact (temperaturelike) critical properties of homogeneous systems (no gradients). Moreover, the conditions under which this is possible can easily be assessed by making explicit some of the ideas that underly the original work of HSL. We will now derive this condition and point out some of its implications.

Again we first recapitulate a result of HSL. By using the dual symmetry of the DRSRG equations for the triangular Ising model with interaction parameters $K_{1}, K_{2}$, and $K_{3}$, HSL could show that the subspace of functions 


$$
\begin{gathered}
C \equiv\left\{\left(K_{1}(\overrightarrow{\mathbf{r}}), K_{2}(\overrightarrow{\mathrm{r}}), K_{3}(\overrightarrow{\mathrm{r}})\right) \mid \sinh \left[2 K_{1}(\overrightarrow{\mathrm{r}})\right] \sinh \left[2 K_{2}(\overrightarrow{\mathrm{r}})\right]+\sinh \left[2 K_{2}(\overrightarrow{\mathrm{r}})\right] \sinh \left[2 K_{3}(\overrightarrow{\mathrm{r}})\right]\right. \\
\left.+\sinh \left[2 K_{3}(\overrightarrow{\mathrm{r}})\right] \sinh \left[2 K_{1}(\overrightarrow{\mathrm{r}})\right]=1\right\}
\end{gathered}
$$

was invariant under the flow. This means that if $K \equiv\left(K_{1}(\vec{r}), K_{2}(\vec{r}), K_{3}(\vec{r})\right) \in C$ initially, than $K \in C$ at all later times. Since the homogeneous triangular Ising model is critical whenever

$$
\begin{aligned}
\sinh \left(2 K_{1}\right) \sinh \left(2 K_{2}\right)+ & \sinh \left(2 K_{2}\right) \sinh \left(2 K_{3}\right) \\
& +\sinh \left(2 K_{3}\right) \sinh \left(2 K_{1}\right)=1,
\end{aligned}
$$

inhomogeneous triangular lattices for which $K \in C$ are "locally critical"2 everywhere; HSL therefore associated $C$ with the critical subspace of their flow equations. Similar subspaces of lattices that are locally critical everywhere were found in all other applications of DRSRG. We therefore now assume that whenever one can apply DRSRG to a given lattice model which is known to be critical if $c\left(K_{1}, \ldots, K_{n}\right)=0$, then one can also show that the subspace

$$
C \equiv\left\{\left(K_{1}(\overrightarrow{\mathrm{r}}), \ldots, K_{n}(\overrightarrow{\mathrm{r}})\right) \mid c\left(K_{1}(\overrightarrow{\mathrm{r}}), \ldots, K_{n}(\overrightarrow{\mathrm{r}})\right)=0\right\}
$$

is an invariant subspace of the flow equation (10). $C$ is associated with the critical subspace of Eq. (10), and fixed-point solutions will be supposed to lie in C.

Let us consider at fixed position $\overrightarrow{\mathrm{r}}$ the vector $\xi_{i}$ orthogonal to the surface $c\left(K_{1}(\overrightarrow{\mathrm{r}}), \ldots, K_{n}(\overrightarrow{\mathrm{r}})\right)=0$,

$$
\left.\xi_{i} \equiv \frac{\partial c}{\partial K_{i}}\right|_{c=0}
$$

It is easy to show ${ }^{19}$ that the assumption that $C$ is invariant under the flow (10) implies that $\xi_{i}$ is a left eigenvector of $\overrightarrow{\mathrm{D}}_{i j}$,

$$
\begin{aligned}
& \sum_{i=1}^{n} \xi_{i}\left(K_{1}, \ldots, K_{n}\right) \overrightarrow{\mathrm{D}}_{i j}\left(K_{1}, \ldots, K_{n}\right) \\
& =\vec{\mu} \xi_{j}\left(K_{1}, \ldots, K_{n}\right) \text { for all } K \in C .
\end{aligned}
$$

Since $\xi_{i}$ and $\overrightarrow{\mathrm{D}}_{i j}$ are in any application known functions, $\vec{\mu}$ can, according to (11), be calculated as the eigenvalue of the left eigenvector $\xi_{i}$ of the finite matrix $\overrightarrow{\mathrm{D}}_{i j}$. Moreover, we see that $\vec{\mu}$ cannot depend on $\overrightarrow{\mathrm{r}}$ explicitly since neither $\xi_{i}$ nor $\overrightarrow{\mathrm{D}}_{i j}$ does. Hence $\vec{\mu}=\vec{\mu}\left(K_{1}(\vec{r}), \ldots, K_{n}(\vec{r})\right)$, an explicit function only of the $K_{i}$.

The linearized flow equation for arbitrary perturbations $\delta K_{i}$ around an arbitrary fixed point $K_{i}^{*}(\overrightarrow{\mathrm{r}})$ reads

$$
\begin{aligned}
\frac{\partial \delta K_{i}}{\partial t}= & \sum_{j, k}\left(\frac{\partial \vec{D}_{i j}}{\partial K_{k}}\right)^{*} \cdot \vec{\nabla} K_{j}^{*} \delta K_{k} \\
& +\sum_{j}\left(D_{i j}^{*}-\delta_{i j} \overrightarrow{\mathrm{r}}\right) \cdot \vec{\nabla} \delta K_{j} .
\end{aligned}
$$

The critical properties at any fixed point are governed by the flow away from criticality, i.e, the flow in the direction of $\xi_{i}^{*}$. For this component of the flow we find from Eq. (12),

$$
\begin{aligned}
\sum_{i=1}^{n} \xi_{i}^{*} \frac{\partial \delta K_{i}}{\partial t}= & \sum_{i, j, k} \xi_{i}^{*}\left(\frac{\partial \overrightarrow{\mathrm{D}}_{i j}}{\partial K_{k}}\right)^{*} \cdot \vec{\nabla} K_{j}^{*} \delta K_{k} \\
& +\sum_{i j} \xi_{i}^{*}\left(\overrightarrow{\mathrm{D}}_{i j}^{*}-\overrightarrow{\mathrm{r}} \delta_{i j}\right) \cdot \vec{\nabla} \delta K_{j} \\
= & \sum_{i, j, k} \xi_{i}^{*}\left(\frac{\partial \overrightarrow{\mathrm{D}}_{i j}}{\partial K_{k}}\right)^{*} \cdot \vec{\nabla} K_{j} \delta K_{k} \\
& +\sum_{j} \xi_{j}^{*}(\vec{\mu} * \overrightarrow{\mathrm{r}}) \cdot \vec{\nabla} \delta K_{j},
\end{aligned}
$$

where we used Eq. (11) in the second line. Equation (13) shows that the linear flow away from criticality depends in general on the gradients $\vec{\nabla} \delta K_{i}$ and is therefore nonuniversal. However, it is independent of the gradients if and only if there is a fixed-point solution satisfying

$$
\vec{\mu}\left(K_{1}^{*}(\overrightarrow{\mathrm{r}}), \ldots, K_{n}^{*}(\overrightarrow{\mathrm{r}})\right)=\overrightarrow{\mathrm{r}} \text { for all } \overrightarrow{\mathrm{r}} .
$$

In this case the first term on the right-hand side of Eq. (13) gives an explicit expression for the thermal eigenvalue $y_{T}$ [Eq. (6.11) of HSL] which can be interpreted as the eigenvalue of the homogeneous lattice.

Equation (14) is an important relation: Not only does it enable us to investigate the general properties of the DRSRG flow equations, but it also simplifies the calculations enormously in a given application. For instead of solving the intricate partial differential equations for the fixed-point solution obtained by putting the left-hand side of Eq. (10) equal to zero, we may directly arrive at the useful fixed-point solution by solving the algebraic equations (14) together with

$$
c\left(K_{1}^{*}(\overrightarrow{\mathrm{r}}), \ldots, K_{n}^{*}(\overrightarrow{\mathrm{r}})\right)=0 .
$$

The latter equation expresses the fact that $K^{*}$ should lie in the critical subspace $C$.

However, besides simplifying the mathematics, 
the above procedure to obtain the proper fixed-point solution by a short cut has interesting conceptual implications too. Since $\vec{\mu}$ does not depend on $\vec{r}$ explicitly, Eqs. (13) and (14) always constitute $d+1$ independent equations, where $d$ is the spatial dimension. Obviously, for these equations to be solvable, it is necessary that $n \geq d+1$. Thus in DRSRG theory one needs at least $d+1$ parameters in order to compute the thermal eigenvalue of a $d$ dimensional lattice model. This result indicates that the "decoration" transformation, ${ }^{3}$ involving two parameters, is in a sense the "natural" transformation for DRSRG in one dimension, ${ }^{13}$ and the STT is the "natural" one in two dimensions. In higher dimensions, appropriate generalizations of the STT seem necessary. Indeed, precisely such generalizations were invented by Yamazaki et al. ${ }^{6}$ to apply DRSRG to the Gaussian model in arbitrary dimensions. For other lattice models, however, such generalizations are not known.

Although we are led again to the conclusion that the STT is an essential tool in the present formulation of DRSRG, there remain several questions to be answered; e.g., can the basic property (14) be related to any known property of homogeneous Ising models? Moreover, why can this relation be obtained irrespective of any knowledge of the boundary conditions for the functions $K_{i}(\vec{r})$ ?

While we have shown in this section under what conditions the linear flow away from criticality can be independent of the gradients, not much is known about the question for which properties this line of analysis can be followed. To our knowledge it is not clear whether the fact that anisotropy is a marginal operator for isotropic Ising models ${ }^{20}$ has its counterpart in DRSRG. As regards the nonlinear flow properties, it is unlikely that one can associate these with properties of homogeneous systems, since the nonlinear flow will not be independent of the gradients. Indeed, Knops and Hilhorst ${ }^{21}$ studied the nonlinear flow in the critical subspace of the triangular Ising model, but were unable to relate their findings to known properties of homogeneous Ising models. It may therefore well be that the assumption leading to Eq. (7) for the correlation function is only justified for the linear flow away from criticality at the fixed point given by Eqs. (14) and (15).

Finally, we ought to mention that Stella ${ }^{22}$ has shown how the Migdal-Kadanoff transformation ${ }^{23}$ in the $l \rightarrow 1$ limit can be interpreted in the light of the ideas of HSL. In that case bonds are redistributed uniformly over the lattice, so that no inhomogeneities develop. Our remarks therefore do not pertain to such cases. We think, however, that our analysis is relevant if one tries to turn DRSRG into an approximate theory in which inhomogeneities still occur, since a requirement equivalent to Eq. (14) must hold even in such cases.

\section{ACKNOWLEDGMENT}

I am grateful to John D. Weeks for comments on the manuscript.
*Present address.

${ }^{1}$ Th. Niemeijer and J. M. J. van Leeuwen, in Phase Transitions and Critical Phenomena, edited by C. Domb and M. S. Green (Academic, New York, 1976), Vol. 6.

${ }^{2}$ H. J. Hilhorst, M. Schick, and J. M. J. van Leeuwen, Phys. Rev. Lett. 뜨, 1605 (1978); Phys. Rev. B $\underline{19}, 2749$ (1979).

${ }^{3}$ For a review, see I. Syozi, in Phase Transitions and Critical Phenomena, edited by C. Domb and M. S. Green (Academic, New York, 1972), Vol. 1.

${ }^{4}$ Y. Yamazaki and H. J. Hilhorst, Phys. Lett. 70A, 329 (1979).

${ }^{5}$ Y. Yamazaki, H. J. Hilhorst, and G. Meissner, Z. Phys. B 35, 333 (1979).

${ }^{6}$ Y. Yamazaki, H. J. Hilhorst, and G. Meissner, J. Stat. Phys. 23, 609 (1980).

7J. M. J. van Leeuwen, in Perspectives in Statistical Physics, edited by H. J. Raveché (North-Holland, Amsterdam, 1981).

${ }^{8}$ W. van Saarloos, Physica 112A, 65 (1982).

${ }^{9}$ W. Jezewski, Phys. Rev. B 24, 3984 (1981).

10J. M. J. van Leeuwen, in Fundamental Problems in Sta- tistical Mechanics III, edited by E. G. D. Cohen (North-Holland, Amsterdam, 1975).

${ }^{11}$ More extensive introductions to the main ideas of the theory can be found in Refs. 2, 7, and 12.

${ }^{12}$ H. J. Hilhorst and J. M. J. van Leeuwen, Physica 106A, 301 (1981).

${ }^{13}$ W. van Saarloos, J. M. J. van Leeuwen, and A. L. Stella, Physica 97A, 319 (1979).

${ }^{14}$ More precisely, under an inversion of the coordinates $(\vec{r} \rightarrow-\vec{r})$, the lattice sites indicated with open circles go over into lattice sites indicated by solid circles and vice versa. For this reason there are no groups of spins on the sublattice of open circles whose center coincides with the origin.

${ }^{15}$ A. Pais, Proc. Natl. Acad. Sci. U.S.A. 40, 34 (1963).

16F. J. Wegner, J. Math. Phys. 12, 2259 (1971).

${ }^{17}$ The analysis of Ref. 9 appears to be based on the observation that if all interactions connecting spins at the edges of $L_{s}$ to spins in the bulk of $L_{s}$ are identically zero, the transformation to the lattice of Fig. 3(d), of course, exists: The latter is then just the bulk part of the former. However, by requiring that this type of 
transformation is repeatable $1,2,3,4, \ldots$ times, it then follows that the $1,2,3,4, \ldots$ outer rows of interactions, and thus all the interactions of $L_{s}$ must be identically zero. In that case the renormalization-group transformation does not contradict Eq. (7), but is a trivial transformation for uncoupled spins. The above use of boundary conditions should not be confused with those of HSL (Sec. II). Theirs are imposed to ensure that the flow is infinitesimal near the boundaries but are not necessary to make the transformation of Fig. 2 work.

${ }^{18}$ The flow equations are somewhat different if the transformation involves auxiliary parameters. As shown explicitly in Ref. 8, however, our line of argument can be extended to such cases.

${ }^{19}$ The proof for general $n$ is exactly the same as the one given for $n=3$ by HSL in Ref. 2, Eqs. (4.20)-(4.22).

${ }^{20}$ L. P. Kadanoff and H. Ceva, Phys. Rev. B $\underline{3}, 3918$ (1971).

${ }^{21}$ H. J. F. Knops and H. J. Hilhorst, Phys. Rev. B $\underline{19}$, 3689 (1979).

${ }^{22}$ A. L. Stella, Physica 111A, 513 (1982).

${ }^{23}$ L. P. Kadanoff, Ann. Phys. (N.Y.) 100, 359 (1976). 